\title{
The Etiology of COVID-19 in Silico by SARS-Cov-2 Infection with the Quantum Microrna Language-AI
}

\author{
Fujii YR* \\ Kawada-Cho, 106-6, Atsuta-Ku, Nagoya, Japan
}

*Corresponding author: Yoichi Robertus Fujii, Kawada-Cho, 106-6, Atsuta-Ku, Nagoya, Japan, Tel: 81526827003; Email: fatfuji@hotmail.co.jp

\section{Research Article}

Volume 4 Issue 2

Received Date: May 11, 2020

Published Date: July 02, 2020

DOI: $10.23880 / v i j-16000243$

\section{Abstract}

Objective: Coronavirus disease 2019 (COVID-19) is complexed infectious disease caused by severe respiratory syndrome (SARS) human coronavirus 2 (CoV-2). We have previously shown that the microRNA (miRNA) entangling sorter (METS) analysis with quantum miRNA/miRNA language is available for the etiology investigation in silico of human virus-associated diseases. To investigate COVID-19 etiology, SARS-CoV-2 infection was simulated by METS algorithm with artificial intelligence (AI) machine learning (MIRAI).

Materials and Methods: The information of coronavirus was extracted from database. Putative CoV-2 miRNAs were predicted by functionally analogy analysis. Statistical data was calculated by Prediction One.

Results: The quantum miRNA immunity was observed in SARS-CoV-2 infection. Acute inflammation and viral infection mechanisms in COVID-19 were independently shown in host and viral miRNA networks according to the output of MIRAI. SARS-CoV-2 infection induced IL-6 upregulation by downregulation of miR-98-5p hub, and hypoxia was induced protein HIF1A suppression by viral miRNAs. C1q complement inhibition was tuned by viral miRNAs.

Conclusion: We found in silico that COVID-19 might show IL-6 production by host miRNAs, and hypoxic vascular hypertension and hypocomplementemia-like symptom by viral miRNAs.

Keywords: Microrna; SARS-Cov-2; Fibrosis; Quantum Microrna Language; Quantum Microrna Immunity; Machine Learning, AI; VR, Covid-19; Pneumonia, Inflammation

\section{Introduction}

Since December on 2019, the zoonotic human coronavirus (CoV) pandemic has emerged by severe acute respiratory syndrome (SARS) CoV-2, and SARS-CoV-2related disease was established as coronavirus disease 2019 (COVID-19) (World Health Organization, WHO) (www. who.int) by the International Committee on Taxonomy of Viruses (ICTV) [1]. On 30 ${ }^{\text {th }}$, January 2020, WHO declared the novel coronavirus, SARS-Cov-2 outbreak as a public health emergency of international concern (PHEIC). At $1^{\text {st }}$ May, 2020, 3,181,642 infections and 224,301 death in the world were confirmed by WHO. Clinical manifestations in this lethal disease at first are pneumonia, fever, difficulty of breathing, and the computed tomography (CT) of bilateral lower lung lobes with vascular dilatation and the halo sign. Given the data of the bed side, approximately $17.7-32.0 \%$ of severe individuals need a care of intensive care unit (ICU) [2]. Further, cytokine releasing syndrome (CRS) in the severe COVID-19 patients was observed, such as high level of interleukin-6 (IL-6), interleukin-1 $\beta$ (IL-1 $\beta$ ), induced protein 10 (IP10) and monocyte chemoattractant protein-1 (MCP-1) in patients' sera [3,4] and severe lymphopenia [5]. It is well known that IL-6 is secreted from macrophages including alveolar macrophages, $\mathrm{T}$ and $\mathrm{B}$ lymphocytes, fibroblast endothelial cells and mesangial cells, and IL- 6 is also a 


\section{Virology \& Immunology Journal}

central mediator of fever and the acute phase inflammation [6]. In an experiment in vivo of mouse pneumonia virus infection model, ablation of IL-6 producing alveolar macrophage resulted prolonged survival of infected mice [7]. In the administration, tocilizumab, humanized anti-IL-6 receptor monoclonal antibody, was an effective treatment in severe patients of COVID-19 [8]. Corticosteroids, one of anti-inflammatory agents, were commonly prescribed in the treatment of COVID-19 patients [9]. It is suggested that cytokine storm by IL- 6 dysregulation may cause lung injury of COVID-19. However, the precious mechanism of the CRS in virus/host interaction remains unclear.

Complement $\mathrm{C} 1 \mathrm{q}$ is a starting molecule of the classical complement pathway, which quickly initiates $\mathrm{C}_{1 \mathrm{qC}} 1 \mathrm{r}_{2} \mathrm{C} 1 \mathrm{~s}_{2}$ complex. The complex recognizes the Fc portion of IgM and IgG antibodies binding with antigen and the complement cascade with C3, C5, C6, C7, C8 and C9 induces cytolytic effects to virus infected cells [10]. C1q-deficiency in humans causes development of systemic lupus erythematosus (SLE) and SLE-like hypocomplementemia [11]. Since C1q enhanced Fc $\gamma$ R-mediated phagocytic function in macrophages as opsonization, C1q-deficiency reduced clearance of an immune-complex. Therefore, SLE-like glomerulonephritis was induced by an immune-complex. COVID-19 patients showed kidney injury by the immune complex [12]. However, while SLE is characterized as autoimmune disease by autoantibodies and there is no evidence of autoantibodies in COVID-19, the etiology of SLE is enough to be distinct from that of CRS in the clinical data of COVID-19. In case reports of virus infection, hepatitis C virus (HCV) was complicated with C3 and C4 hypocomplementemia and increasing risk of thrombosis was observed in a human immunodeficiency virus type 1 (HIV-1) infected patient with SLE [13,14]. Furthermore, parvovirus B19 infection had similar symptoms of SLE and the infected patients showed low serum levels of $\mathrm{C} 3$ and C4 [15]. On the other hand, in clinical active SLE, IL-6 and circulating immune complexes were significantly increasing and immune regulatory lectin IL-6 increases complement factor B to induce the complement alternative pathway for acute inflammation in hepatocytes and human alveolar type II epithelial cells [16-18]. Although a COVID-19 patient complicated with thrombosis anti-SLE agent, hydroxychloroquine would not be effective to suppress IL-6 production $[19,20]$. Thus, it is suggested that three key subjects, complement, IL-6 and SLE-like symptoms into acute inflammation may be involved into CRS of COVID-19 under lethal pneumonia; however, the etiology of COVID-19 has not yet been established.

SARS-CoV-2 targets angiotensin converting enzyme 2 (ACE2), which are expressed on various cells, such as lung, heart, kidney and gastrointestinal tract cells [21]. Viral diameter enveloped is $65-125 \mathrm{~nm}$ containing single plus strand of RNA. About host-virus interaction in immune system, pathogenicity of viral protein has been reported. Wild type SARS-CoV non-structural protein NSP3 suppressed type 1 interferon (IFN), interferon-stimulated gene 15 (ISG15), c-X-c motif chemokine ligand 10 (CXCL10), IL-6 and tumor necrosis factor (TNF) in mouse model, but lung pathology development was observed. In contrast, mutant of NSP3 attenuated severe respiratory disease and prolonged survival time, but proinflammatory cytokines were increased [22]. Although the data is obtained by SARS-CoV, IL-6 production did not cooperate to express severe pneumonia even though NSP3 had virulence against the mouse lung. Therefore, it is remained to elucidate pathogenicity of SARS-CoV infection in humans.

The host microRNA (miRNA) controls pathogenicity of viruses, such as hepatitis B virus (HBV), HCV and HIV$1[23,24]$. We have been reported that miRNA biomarker panels show the etiology of viral infection, which was in silico simulated by miRNA entangling target sorting (METS) algorithm with artificial intelligence (AI) machine learning (MIRAI) [24]. Subsequently, we found the third host defense neo-mechanism, 'the quantum miRNA immunity' against human hepatitis and immunodeficiency viruses. In the computer simulation, viral miRNAs had an important role to develop pathogenicity of viruses. To further investigate the etiology of the quantum miRNA immunity against SARS$\mathrm{CoV}$ infection, the miRNA profiles of SARS-CoV-infected alveolar stem cells and SARS-CoV-2 miRNA candidates were computed by the METS analysis with AI.

\section{Materials and Methods}

\section{Database Usage}

Google scholar (https://scholar.google.co.jp) and PubMed (www.ncbi.nlm.gov/pubmed/) were used for extraction of miRNA panel data. Total information content was 20,735 in coronavirus infection. The gene function of protein was searched by GeneCards (www.genecards. org). Protein ontology was investigated by GO enrichment analysis in Geneontology (geneontology.org). Data mining about miRNA profile was performed by cleared in top four expression levels of up- and down-regulation.

\section{METS in silico Analysis}

MMP calculation and METS analysis were performed by the computer processing as described previously [23, 24-28]. MMP from miRNA biomarker panels was calculated by double miRNAs' quantum energy levels of double nexus score (DNS) entangling single miRNA quantum energy 


\section{Virology \& Immunology Journal}

levels of single nexus score (SNS) (Table 1). Data of multitargets to a miRNA were extracted from TargetScan Human 7.2 (targetscan.org) and miRTarBase Ver. 8.0 (mirtarbase. cuhk.edu.cn). Target protein/protein interaction and cluster analysis was searched by STRING Ver. 11.0 (string-db.org).

\begin{tabular}{|c|c|c|c|c|c|}
\hline Host miRNA & Level & SNS & Viral miRNA & Level & SNS \\
\hline miR-331-3p & up & 5 & COV-miR-1 & up & 4 \\
\hline miR-497-5p & up & 6 & COV-miR-2 & up & 5 \\
\hline miR-574-5p & up & 11 & COV-miR-4 & up & 3 \\
\hline miR-106a-5p & down & 7 & COV-miR-5 & up & 8 \\
\hline miR-186-5p & down & 4 & COV-miR-6 & up & 8 \\
\hline miR-223-5p & down & 6 & & & \\
\hline miR-146b-5p & down & 5 & & & \\
\hline miR-98-5p & down & 7 & & & \\
\hline
\end{tabular}

Table 1: Host and viral miRNAs in the simulation.

\section{Viral Mirna and Functionally Analogy Analysis}

Data of viral miRNA and viral genome was obtained from Viral Genomes (ncbi.nim.nih.gov) and miRBase Ver. 22.1 (miRbase.org). SARS-CoV-2 sequence (NC_045512.2) was used for prediction of putative viral miRNA in the 3'UTR. The functional analogy between viral miRNA and host miRNA was performed as previously described [24,29,30]. MiRCompare (160.80.35.140) was used for homology sequence search. The RNA secondary structure of RNA was computed by RNA Folding Form (unafold.rna.albany.edu).

\section{AI Machine Learning}

Prediction One Ver. 04.08.20 (Sony Network communications Inc. Tokyo, Japan) was used for AI machine learning. The area under the curve (AUC) in receiver operating characteristic (ROC), accuracy, precision and F values were calculated as the percentage by Prediction One.

\section{MIRAI}

Previous METS analysis data in hepatitis B virus (HBV), hepatitis $\mathrm{C}$ virus (HCV) and human immunodeficiency virus type 1 (HIV-1) infection was combined with the present data to produce the AUC data through AI machine learning.

\section{Results and Discussion}

\section{METS Analysis with MIRAI}

METS analysis was performed by AI in SARS-CoV-2 infection. Since the miRNA profile of SARS-CoV infection has been shown in bronchoalveolar stem cells the miRNA profile data as single miRNA panel was mining and applied for METS analysis because generally the cellular miRNA profile has the similarity with circulating miRNA one in exosome and miRNAs in extracellular vesicles constitute a communication among cells [31-33]. We selected three upregulated host miRNAs, miR-331-3p, miR-497-5p and miR-574-5p, and four downregulated host miRNAs, miR-106a-5p, miR-1865p, miR-223-5p, miR-146b-5p and miR-98-5p (Table 1). In miRNA target and protein/protein cluster of the quantum energy levels, miR-331-3p, miR-186-5p, miR-223-5p and miR-146b-5p were less data to be applied for METS analysis with AI (data not shown).

SARS-CoV-2 encoded miRNAs have been identified in silico $[34,35]$. Coronavirus miRNA was also encoded in the genome of Middle East respiratory syndrome (MARS)-CoV [36]. SARS-CoV was encoded viral small RNAs from the NSP3 region and the $\mathrm{N}$ region (svRNA-N), and anti-svRNA-N reduced lung pathogenic effects in vivo [37]. From these evidences, we predicted SARS-CoV-2 miRNA candidates from two stem loops in the 3' terminal and from the 3'UTR region because functional RNA viral miRNAs have been encoded in the non-coding region of the 3' end [23] (Figue 1). To cohere SARS-CoV-2 miRNA candidates in the METS analysis, the seed paralogue of viral miRNA candidates was searched in that of human host miRNAs. Cov-miR-1, Cov-miR-2, Cov-miR-4, CovmiR- 5 and Cov-miR- 6 were the seed paralogue of miR-4310 (75\% homologous), miR-4291 and miR-3611 (75\%), miR576-3p (88\%), miR-27a/b-3p (75\%), miR-216a-3p and miR$29 \mathrm{c}-5 \mathrm{p}(75 \%)$, respectively. In the quantum energy levels, Cov-miR-1 was less target and subject data to be applied for the METS analysis with AI (data not shown). 


\section{Virology \& Immunology Journal}

\begin{tabular}{|c|c|c|c|c|}
\hline $\begin{array}{c}\text { Viral miRNA } \\
\text { candidate }\end{array}$ & Viral miRNA sequence & $\begin{array}{c}\text { Analogous human } \\
\text { miRNAS }\end{array}$ & Human miRNA sequence & \%homology* \\
\hline COV2-miR-1 & GCAGAAUGAAUUCUCGU & hsa-miR-4310 & GCAGCAUUCAUGUCCC & 75 \\
\hline COV2-miR-2 & UUGUGCAGGAAUGAAUUCUCGU & hsa-miR-4291 & UUCAGCAGGAAAGCU & 75 \\
\hline COV2-miR-2 & UUGUGCAGAAUGAAUUCUGGU & hsa-miR-3611 & UUGUGAAGAAAGAAAUUCUUA & 75 \\
\hline & UUGUGCAGAAUGAAUUCUGGU & hsa-miR-4520-2-3p & UUGGACAGGAAACACGCAGGU & 38 \\
\hline & UUCUGGUAACUACAUAGC & hsa-miR-4755-5p & UUUCCCUUCAGAGCCUGGUUU & 50 \\
\hline Cov2-miR-4 & UAGAUGUAGUAACUACAUAGC & hsa-miR-5006-3p & UUUCCCUUCAGAGCCUGGCUUU & 50 \\
\hline Cov2-miR-5 & UUACACCGAGGCCACGCGAUACG & hsa-miR-27a-3p & UUCACAGUGGCUAAGUUCCGC & 75 \\
\hline COV2-miR-6 & UCACCGAGGCCACGCCGGAGUACG & hsa-miR-216a-3p & UACACAGUGGUCUCUGGGAUAU & 75 \\
\hline COV2-miR-5 & UUCACCGAGGCCACGCGCGGAGUACG & hsa-miR-27b-3p & UUCACAGUGGCUAAGUUCUGU & 75 \\
\hline COV2-miR-6 & UCACCGAGGACGGCCACGGAGUACG & hsa-miR-290-5p & UGACCGAUUUCUCCUGGUGUUC & 75 \\
\hline
\end{tabular}

Table 2: Viral miRNA seed paralogue in human miRNAs ( ${ }^{*} 8$ seed).

The relation between acute inflammation and viral infection were investigated with host miRNA data and SARSCoV-2 miRNA data by MIRAI (Table 3, Figure 1). Four SARS-
CoV-2 miRNA candidates from the 3' end of the SARS-CoV-2 genome were used for the computing with four host miRNAs (Table 1, Figure 2).

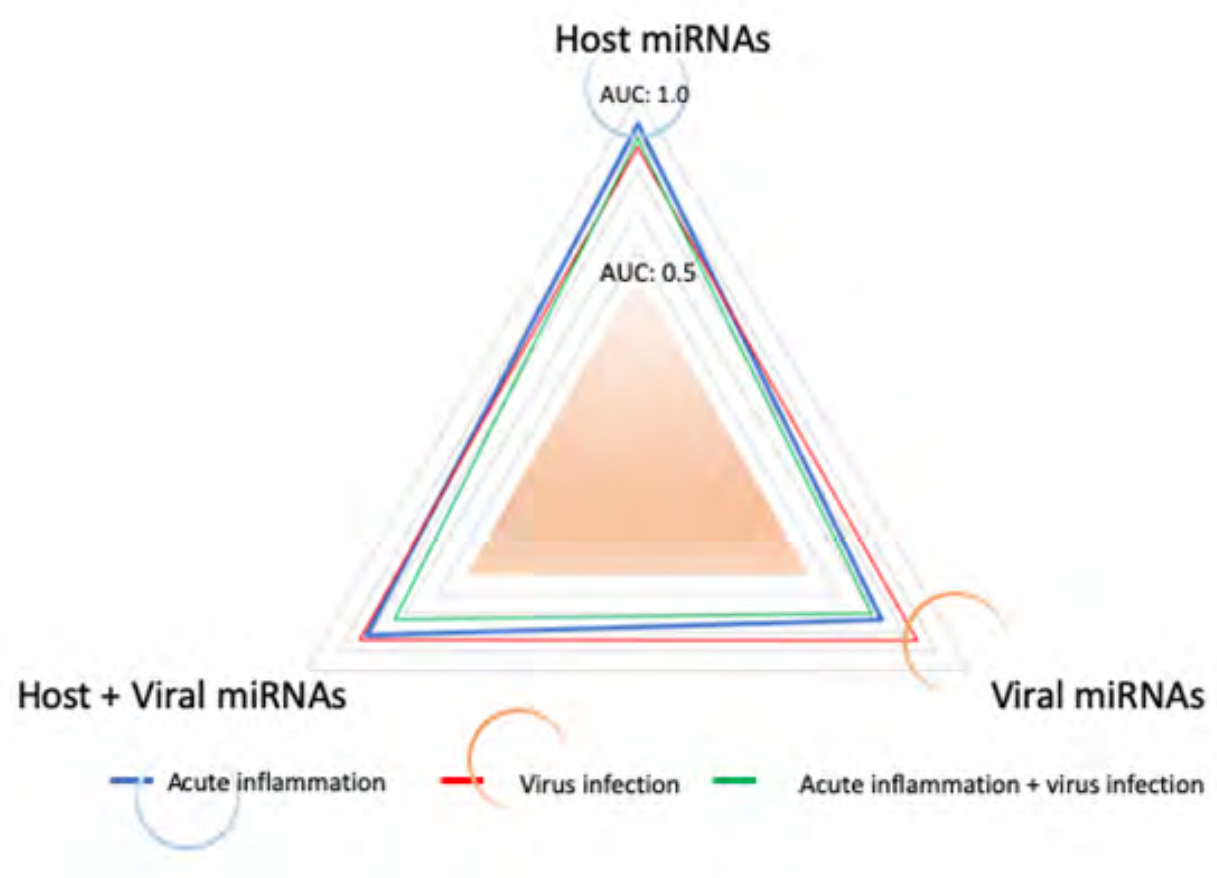

Figure 1: METS analysis with MIRAI. METS analysis against acute inflammation and viral infection was performed by AI machine learning. AUC was calculated in acute inflammation, viral infection, inflammation \& infection with host miRNAs, viral miRNA and host miRNAs plus virus-miRNAs, and the results were depicted with the rad. 


\section{Virology \& Immunology Journal}

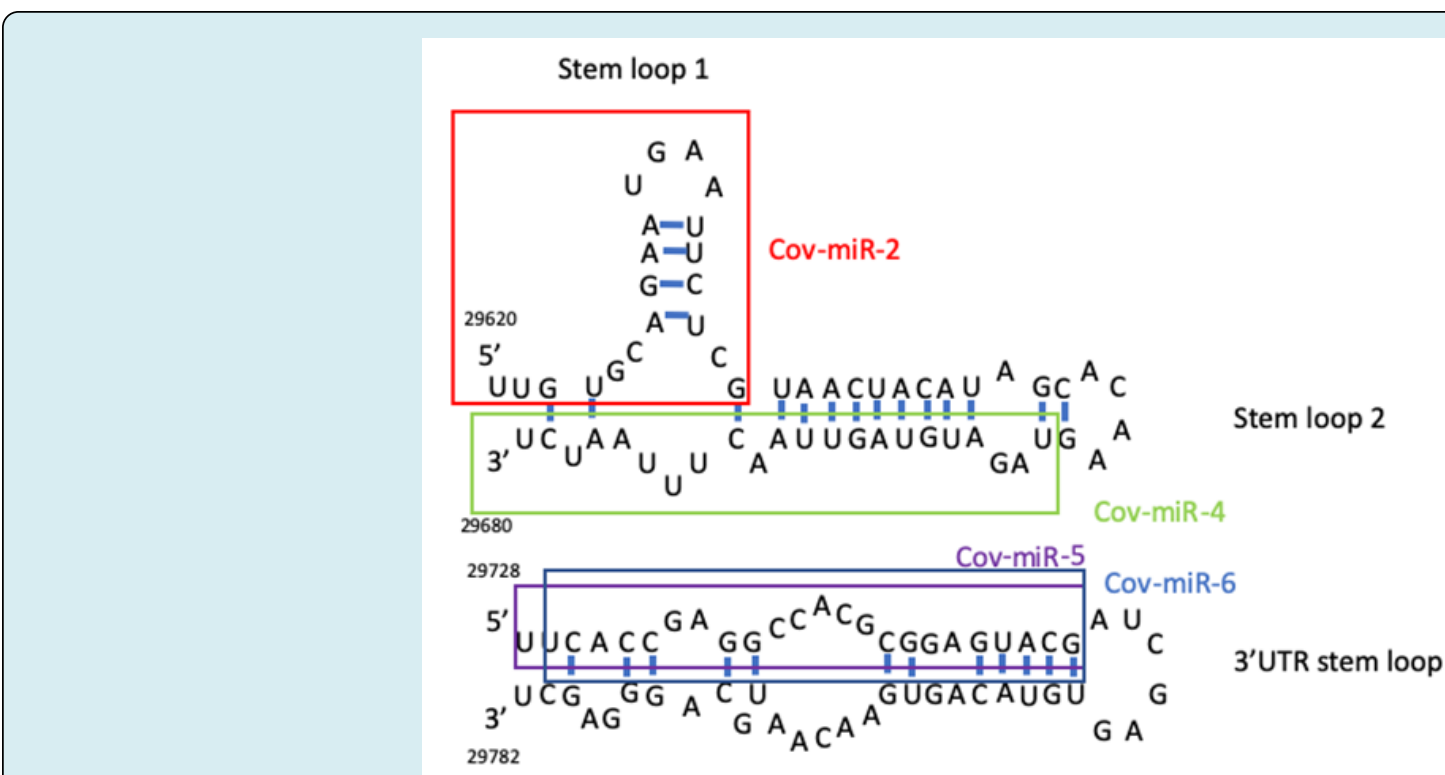

Figure 2: Putative CoV-2 microRNAs. CoV-2-miRNA candidates were presented in the 3' end of the SARS-CoV-2 RNA genome.

The AUC, accuracy, precision, and F values were computed in host miRNA alone, viral miRNA alone and cohered host plus viral miRNAs by MIRAI (Table 3). As shown in Figure 1 , significant AUS value was extracted from host miRNAs with acute inflammation (blue circle) and viral miRNAs with virus infection (red circle). It is suggested that SARS-CoV-2 infection would not be cooperated to acute inflammation by the quantum miRNA immunity. In turn, clinical symptom of COVID-19 causes two distinct miRNA packages, which are derived from host miRNAs and viral miRNAs.

\begin{tabular}{|c|c|c|c|}
\hline \multicolumn{5}{|c|}{ Inflammation } \\
\hline & Host miR & Viral miR & Host+Viral miR \\
\hline AUC & 0.9236 & 0.7336 & 0.8147 \\
\hline Accuracy & 0.9000 & 0.7586 & 0.8768 \\
\hline Precision & 0.9804 & 0.8333 & 0.9589 \\
\hline F value & 0.9434 & 0.8511 & Host + Viral miR \\
\hline \multicolumn{5}{|c|}{ Viral infection } \\
\hline \multicolumn{5}{|c|}{ Host miR } & Viral miR & 0.8345 \\
\hline AUC & 0.8618 & 0.8406 & 0.8768 \\
\hline Accuracy & 0.8833 & 0.7586 & 0.9136 \\
\hline Precision & 0.9444 & 0.9000 & 0.9308 \\
\hline F value & 0.9358 & 0.8372 & Host miR + Virus miR \\
\hline \multicolumn{5}{|c|}{ Inflammation + Viral infection } \\
\hline AUC & Host miR & Viral miR & 0.7308 \\
\hline Accuracy & 0.8836 & 0.7029 & 0.8768 \\
\hline Precision & 0.9000 & 0.7586 & 0.9136 \\
\hline F value & 0.9804 & 0.9000 & 0.9308 \\
\hline
\end{tabular}

Data was calculated by Prediction One.

Table 3: Statistic analysis in SARS-CoV-2 infection. 


\section{Network Analysis on Host miRNAs}

To further elucidate the data of MIRAI, the quantum network analysis with host miRNAs was performed (Figure 3). IL-6 was increased by downregulation of miR-98-5p with let-7a-5p, miR-24-3p and miR-155-5p (Figure 3). IL-6 upregulation of cytokine releasing syndrome (CRS) was observed in the severe COVID-19 patients [3,4]. On the other hand, inflammation-related S100 calcium binding protein A12 (S100A12) was reduced by upregulation of miR-574$5 p$ with miR-1224-5p, miR-5889-5p and miR-5004-5p
(Figure 3) and excess inflammation in the lung was inhibited by downregulation of S100A proteins [38]. Furthermore, anti-inflammation related YOD1 deubiquitinase (YOD1) was increased by downregulation of miR-98-5p with miR196a-5p and miR-196b-5p (Figure 3). The deubiquitinating enzyme YOD1 antagonized the ubiquitin ligase TNF receptor associated factor 6 (TRAF6) and blocked IL-1 signaling pathways therefore, IL-6, TNF $\alpha$, and interleukin-8 (IL-8) excess production would be blocked. It is suggested that acute inflammation by SARS-CoV-2 infection would be controlled by host miRNAs.

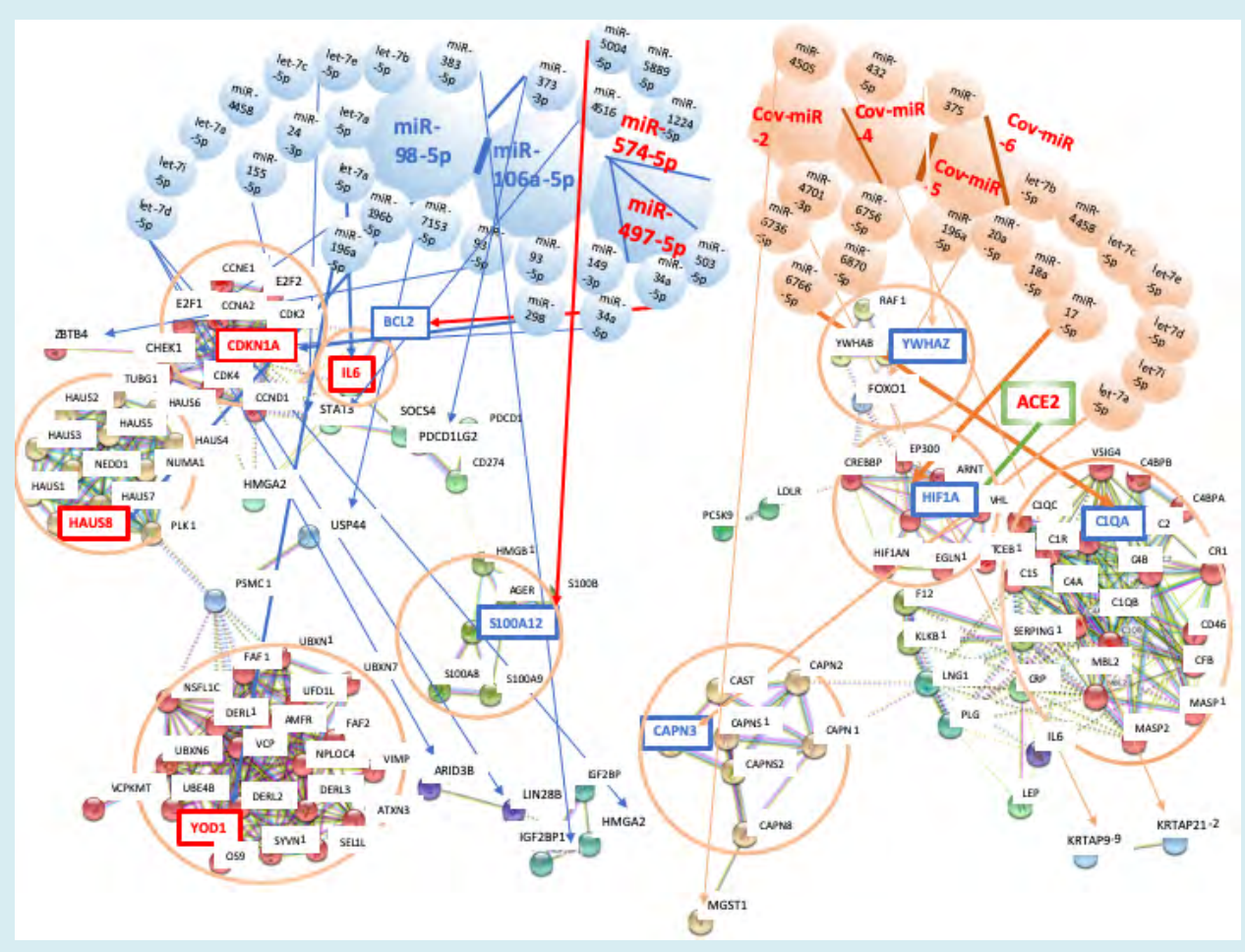

Figure 3: Network analysis of SARS-CoV-2 infection by METS simulation. Network with host miRNAs by METS simulation was presented in the left. SARS-CoV-2 receptor ACE2 was presented in the right green square. Network with viral miRNAs was depicted in the right. miRNAs: blue, downregulation; red, upregulation. Proteins: red, upregulation; blue, downregulation.

HAUS augmin like complex subunit 8 (HAUS8) was augmented by downregulation of miR-98-5p with let-7b$5 p$, let-7e-5p, let-7c-5p, miR-4458, let-7a-5p, let-7i-5p and let-7d-5p (Figure 3). HAUS8 enhances the mitochondrial antiviral signaling protein (MAVS, VISA) [39-41]. RNA virus infection activates the retinoic acid-inducible gene 1 (RIG1) and the activated RIG-1 transduces anti-virus signal via MAVS/VISA to initiate the IKB/NF-kB/interferon regulatory factor 3 (IRF3)/type I interferon (IFN) cascades. HAUS8 reduction by RNA interference inhibited the activation of NF-kB/IRF3/type I IFN cascades [41]. Although we found the quantum immunity in HBV, HCV and HIV-1 infection HAUS8 increasing would show anti-virus effect as the quantum miRNA immunity. Thus, asymptomatic virus carriers were occasionally observed [42]. Concerning cell cycle and apoptosis, anti-apoptotic BCL2 was suppressed by up regulation of miR-497-5p with miR-34a-5p, and cycling dependent kinase inhibitor $1 \mathrm{~A}$ (CDN1A) was augmented by down regulation of miR-106a-5p with miR-149-3p and miR-34a-5p (Figure 3). Therefore, the programmed cell death would be induced in infected lung cells in the quantum miRNA immunity. 


\section{Virology \& Immunology Journal}

\section{Network Analysis on Viral miRNA}

Next, the quantum network analysis with viral miRNA candidates was performed by METS (Figure 3). Hypoxia inducible factor 1 subunit alpha (HIF1A) was reduced by Cov-miR-5 with miR-20a-5p, miR-18a-5p and miR-17-5p (Figure 3). Therefore, oxygen delivery would be decreased and hypoxia would be acutely progressed. Although HIF1A upregulation blocks SARS-CoV-2 receptor ACE2 via let-7 ACE2 expression under the hypoxic condition with pneumonia was kept by Cov-miR-5. SARS-CoV-2 infection would not be reduced even in hypoxic interstitial pneumonia. Hypoxia stimulates the proliferation and migration of pulmonary artery smooth muscle cells (PASMCs) and PASMCs lead to increasing thicking and muscularization of lung vessel walls that is a key of hypoxic pulmonary vascular hypertension in interstitial pneumonia $[43,44]$. Pulmonary vascular hypertension is also controlled by HIF1A expression [45]. The pulmonary hypertension induces reduction of oxygen availability to the lung, for instance, in the case of chronic obstructive pulmonary disease (COPD), idiopathic pulmonary fibrosis (IPF) or pediatric Kawasaki disease [46]. While CovmiR-5 is a seed paralogue of miR-27a/b-3p, miR-27b-5p included into the pre-miRNA of miR-27b-3p was increased both in Kawasaki disease serum and in human umbilical vein endothelial cells of Kawasaki disease [47]. Upregulation of miR-27b-5p inhibited endothelial cell proliferation and migration on Kawasaki disease, but in general, miR-27-5p serves proliferation of the endothelial cells [48]. miR-27b$3 p$ upregulation was implicated in hypoxia, inflammation and apoptosis in idiopathic pulmonary arterial hypertension [49]. Under hypoxic condition with SARS-CoV-2 infection, vascular endothelial cells may be a tendency of apoptotic by Cov-miR-5 and the dead vascular endothelial cells may make blood clots. Together, ACE2 protects against pulmonary hypertension [43] whereas ACE2 is a receptor of SARS$\mathrm{CoV}-2$. The simulation suggested that pulmonary hypoxia and vascular hypertension would be induced by Cov-miR-5, and retained ACE2 expression via suppression of HIF1A would keep viral infection in the lung.

For evasion from the host defense system, Cov-miR-4 blocked complement C1QA with human specific miR-67565p, miR-6870-5p and miR-6766-5p (Figure 3). Deceasing C1q upon the classical pathway in situ abolishes the cytolytic attack with anti-SARS-CoV-2 antibodies against virus producing cells and inhibits opsonization to the immune complex of viruses bound with neutral antibodies or to dead cells which induce thrombosis and SLE-like hypocomplementemia. SARS-CoV-2 specific antibodies/ complement defense system would be stopped but alternative complement pathway activation by IL-6 production via host miR-98-5p downregulation is remained. C3b activation decreases peripheral mononuclear cells (PBMCs), which has been observed in hemodialysis patients [50,51]. Therefore, acute inflammation of non-effective virus eradication might be continued in the lung. If Cov-miR-4 RNAs were stayed into the host cells because complement has an important role for the immunocomplex clearance, SLE-like symptom would be continued for the long-term. This would be one of the immunodeficiency symptoms upon COVID-19. Although convalescent plasma ( $200 \mathrm{ml}$ ) was successfully applied to ten of COVID-19 ICU patients as the transfusion treatment there is some possibility of the effects of neural antibodies plus the complement escaped from incomplete inactivation $\left(30^{\circ} \mathrm{C}\right.$, $4 \mathrm{~h}$ for plasma derivative of hemophilia factor VIII against viruses) because the clinical trial had no blind assessment with inactivated healthy individual plasma. If so, this therapy may be an evidence of the positive effects upon supplement of complementary complement.

Tyrosine 3-monooxygenase/tryptophan 5-monooxygenase activation protein zeta (YWHAZ, 14-3-3 zeta) was inhibited by Cov-miR-5 with miR-375 (Figure 3). Influenza infection specifically induced miR-451 expression and miR-451 suppressed YWHAZ expression, and then IL-6, TNF, c-c motif chemokine ligand 5 (CCL5, RANTES) secretion was inhibited by YWHAZ reduction via forkhead box 03 (FOXO3) upregulation [52,53]. Therefore, YWHAZ is a positive regulator of inflammatory cytokine gene expression. It is suggested that Cov-miR-5 would control IL-6 inflammatory cytokine production for viral infection.

Calcium-activated protease Calpain (CAPN) inhibition has protected from the infection of pathogenic influenza $A$ in vivo and suppresses inflammatory cytokine releasing in the bronchial epithelial cells [54]. Although CAPN3 was inhibited by Cov-miR- 6 with let-7b-5p, let-7e-5p, let-7c-5p, miR-4458, let-7a-5p, let-7i-5pandlet-7d-5p (Figure 3), calpain inhibition by calpain inhibitor MDL28170 suppresses SARS coronavirus replication [55]. Treatment outcome of protease inhibitors, lopinavir/ritonavir combination therapy was useful for patients with SARS-CoV-2 [56]. Therefore, downregulation of calpain would inhibit SARS-CoV-2 excess proliferation. On the contrary, microsomal glutathione S-transferase 1 (MGST1) was decreased by Cov-miR-2 with miR-4505 (Figure 3). Since glutathione S-transferase downregulation was observed in hepatitis D virus infection and its downregulation was implicated in liver injury [57]. MGST1 downregulation may be related with lung injury in SARS-CoV-2 infection. Taken together, Cov-miR-6 would recover the abnormal status of host cells under acute inflammation by host miRNAs for maintaining virus infection and Cov-miR-4 would be a code breaker of host specific immune system against SARS-CoV-2 by destruction of complement classical pathway. Cov-miR-5 would induce pulmonary hypoxia and vascular hypertension. Cov-miR-5 would also retain infectious virus absorption under controlling of acute inflammation by IL- 6 production 


\section{Virology \& Immunology Journal}

via host miR-98-5p downregulation.

From the simulation data by MIRAI, a combination of anti-inflammation and anti-virus agents may be enough necessary for treatment of COVID-19 with the chemotherapeutic and the biologic drugs. In the anti-virus agents, as the 3 ' end noncoding region of the RNA viral genome is generally implicated in viral replication [23], cocktail of miR-98-5p mimic, anti-Cov-miR-4 and anti-CovmiR-5 nucleic acid compounds as the nebulization formula would be possible for development of miRNA vaccine against SARS-CoV-2, which is able to quickly mass-produce with cheap, be easily delivering, virus specific, not borne drug resistant mutants and low side effects [58]. Further data of miRNA biomarker panels from plasma or serum of SARSCoV-2 infection are required for diagnostic and prognostic precious-medicine with MIRAI. We need more progression of in silico investigation to elucidate the etiology of COVID-19 infection and pneumonia.

\section{Conclusion}

We have shown that miRNA profile of SARS-CoV infected bronchoalveolar stem cells would be useful to elucidate the pathogenic machinery of SARS-CoV-2 infection. For the etiology in COVID-19, MIRAI output data that symptom of inflammation was controlled by host miRNAs and that of infection was tuned by viral miRNAs, independently. According to the results of MIRAI computing, in host miRNA network, although the quantum miRNA immunity was observed as cell cycle arrest, apoptosis and an innate immunity of MAVS/VISA, inflammatory IL-6 expression was directly upregulated by downregulation of miR-98-5p hub. On the contrary, HIF1A reduction and C1qA suppression were observed in viral miRNA network. This is the first report that the etiology of COVID-19 was shown from host miRNAs and viral miRNAs independently. The mechanisms upon suppression of hypoxia-related HIF1A protein and complement C1qA by Cov-miR-5 and -4 make hypoxia plus vascular hypertension and an advantage of viral infection while precision of viral miRNA about viral infection was 0.900 in MIRAI. With the host miRNA alone, it was difficult for AI to explain clinical symptoms of COVID-19 at all. Acute inflammation by host miRNAs and low oxygen delivery by viral miRNAs would dominantly cause a lethal and severe pneumonia in COVID-19. As COVID-19 induced IL-6 upregulation by host miR-98-5p hub downregulation, and hypoxia plus vascular hypertension by Cov-miR-5a and complement suppression by Cov-miR-4, subsequently, miR98-5p mimic, anti-Cov-miR-4 and anti-Cov-miR-5 nucleic acid compounds would be candidates of a miRNA therapeutic tool against SARS-CoV-2. Further investigations are needed.

\section{Conflicts of Interest}

The authors declare that there are no conflicts of interest.

\section{References}

1. Coronaviridae Study Group of the International Committee on Taxonomy of Viruses (2020) The species severe acute respiratory syndrome-related coronavirus: classifying 2019-nCoV and naming it SARS-Cov-2. Nat Microbiol 5(4): 536-544.

2. Yang $X, Y u$ Y, Xu J, Shu H, Xia J, et al. (2020) Clinical course and outcomes of critically ill patients with SARSCov-2 pneumonia in Wuhan, China: a single-centered, retrospective, observational study. Lancet in 2600 (20): 30079-30075.

3. Herold T, Jurinovic V, Arnreich C, Hellmuth JC, von Bergwelt Baildon M, et al. (2020) Level of IL-6 predicts respiratory failure in hospitalization symptomatic COVID-19 patients. meDRxiv in press.

4. Gao Y, Li T, Han M, Li X, Wu D, et al. (2020) Diagnostic utility of clinical laboratory data determinations for patients with the severe COVID-19. J Med Virol, pp: 1-6.

5. Wang J, Xu Z, Wang J, Feng R, An Y, et al. (2020) CT characteristics of patients infected with 2019 novel coronavirus: association with clinical type. Clin Radiol 75(6): 408-414.

6. Rose John S (2016) Interleukin-6 family cytokines. Cold Spring Harb Perspect Biol 10(2): a028415.

7. Percopo CM, Ma M, Brenner TA, Krumholz JO, Break TJ, et al. (2019) Critical adverse impact of IL-6 in acute pneumovirus infection. J Immunol 202(3): 871-882.

8. Xu X, Han M, Li T, Sun W, Wang D, et al. (2020) Effective treatment of severe COVID-19 patients with Tocilizumab. Proc Natl Acad Sci USA 117(20): 10970-10975.

9. Wang D, Hu B, Hu C, Zhu F, Liu X, et al. (2020) Clinical characteristics of 138 hospitalized patients with 2019 novel coronavirus-infected pneumonia in Wuhan, China. JAMA 323(11): 1061-1069.

10. Thielens NM, Tedesco F, Bohlson SS, Gaboriaud C, Tenner AJ (2017) C1q: a fresh look upon old molecule. Mol Immunol 89: 73-83.

11. Stegert M, Bock M, Trendelenburg M (2015) Clinical presentation of human C1q deficiency: how much of a lupus. Mol Immunol 67(1): 3-11.

12. Liu B, Li M, Zhou Z, Guan X, Xiang Y (2020) Can we use interleukin-6 (IL-6) blockade for coronavirus disease 


\section{Virology \& Immunology Journal}

2019 (COVID-19)-induced cytokine release syndrome (CRS). J Autoimmun in press.

13. Ray S, Rouse K, Appis A, Novak R, Haller NA (2008) Fibrillary glomerulonephritis with hepatitis C viral infection and hypercomplementemia. Renal Failure 30(7): 759-762.

14. Asherson RA, Gómez Puerta JA, Marinopoulos G (2005) Recurrent pulmonary thromboembolism in a patient with systemic lupus erythematosus and HIV-1 infection associated with the presence of antibodies to prothrombin: a case report. Clin Infect Dis 41(10): e8992.

15. Hashizume H, Kageyama R (2017) Hypocomplementemia is a diagnostic clue for parvovirus B19 infection in adults. J Dermtol 44(3): e27.

16. Thanadetsuntorn C, Ngamjanyaporn P, Sethaudom C, Hodge K, Saengplya N, et al. (2018) The model of circulating immune complexes and interleukin-6 improves the prediction of disease activity in systemic lupus erythematosus. Sci Rep 8(1): 2620.

17. Morrone G, Ciliberto G, Oliviero S, Arcone R, Dente L, et al. (1988) Recombinant interleukin- 6 regulates the transcriptional activation of a set of human acute phase genes. J Biol Chem 263(25): 1554-12558.

18. Zhao YX, Andoh A, Shimada M, Takaya H, Hata K, et al. (2000) Secretion of complement components of the alternative pathway ( $\mathrm{C} 3$ and factor $\mathrm{B}$ ) by the human alveolar type II epithelial cell line A549. Int J Mol Med 5(4): 415-419.

19. de Barry O, Mekki A, Diffre C, Seror M, Hajjan ME, et al. (2020) Arterial and venous abdominal thrombosis in a 79-year-old woman with COVID-19 pneumonia. Radiol Case Rep in press.

20. Goyal M (2020) SLE patients are not immune to covid-19: importance of sending the right message Across. Ann Rheum Dis.

21. Astuti I, Ysrafil (2020) Severe acute respiratory syndrome coronavirus 2 (SARS-CoV-2): an overview of viral structure and host response. Diabet Metab Synd Clin Res Rev 14(4): 407-412.

22. Fehr AR, Channappanavar R, Jankevicius G, Fett C, Zhao J, et al. (2016) The conserved coronavirus macrodomain promotes virulence and suppresses the innate immune response during severe acute respiratory syndrome coronavirus infection. mBio 7(6): e01721-1716.

23. Fujii YR (2017) The microRNA 2000: from HIV-1 to healthcare. Scientific Research Publishing Inc Irvine CA.
24. Fujii YR (2020) The quantum microRNA immunity in human virus-associated diseases: virtual reality of HBV, HCV and HIV-1 infection, and hepatocellular carcinogenesis with AI machine learning. Arch Clin Biomed Res 4(3): 89-129.

25. Fujii YR (2013) The RNA gene information: retroelementmicroRNA entangling as the RNA quantum code. Methods Mol Biol 936: 47-67.

26. Yoshikawa M, Osone T, Fujii YR (2016) MicroRNA memory I: the positive correlation between synergistic effects of microRNAs in cancer and a novel quantum scoring system. J Adv Med Phar Sci 5(4): 1-16.

27. Osone T, Yoshikawa M, Fujii YR (2016) MicroRNA memory II: a novel scoring integration mdel for prediction of human disease by microRNA/microRNA quantum multiinteraction. J Adv Med Phar Sci 5(3): 1-18.

28. Fujii YR (2018) Quantum language of microRNA: application for new cancer therapeutic targets. Methods Mol Biol 1733: 145-157.

29. Yoshikawa M, Fujii YR (2016) Human ribosomal RNAderived resident microRNAs as the transmitter of information upon the cytoplasmic cancer stress. Biomed Res Int 2016: 7562085.

30. Fujii YR (2019) Quantum microRNA network analysis in gastric and esophageal cancers: xenotropic plant microRNAs cure from cancerous paradox via Helicobacter pylori infection. Gastroentrrol Hepatol Endosc 4: 1-18.

31. Malick B, Ghosh Z, Chakrabarti J (2009) MicroRNome analysis unravels the molecular basis of SARS infection in bronchoalveolar stem cells. PLoS One 4(11): e7837.

32. Kouwaki T, Okamoto M, Tsukamoto H, Fukushima Y, Oshiumi H (2017) Extracellular vesicles deliver host and virus RNA and regulate innate immune response. Int J Mol Sc 18(3): 666.

33. Yoshikawa FS, Teixeira FM, Sato MN, Oliveira LM (2019) Delivery of microRNAs by extracellular vesicles in viral infections: could the news be packaged? Cells 8(6): 611 .

34. Periwal N, Sarma S, Arora P, Sood V (2020) In-silico analysis of SARS-CoV-2 genome: insights from SARS encoded non-coding RNAs. bioRxiv in press.

35. Pasquier C, Robichon A (2020) SARS-Cov-2 might manipulate against its host the immunity RNAi/Dicer/ Ago system. Do mitochondria collapse upon COVID-19 infection? bioRex in press.

36. Hasan M, Akter R, Ullah S, Abedin J, Ullah A, et al. (2014) A computational approach for predicting role of human microRNAs in MARS-CoV genome. Adv Bioinform, pp: 
$1-8$.

37. Morales L, Oliveros JC, Fernandez-Deigado R, tenOever BR, Enjuanes L, et al. (2017) SARS-CoV-encoded small RNAs contribute to infection-associated lung pathology. Cell Host Microbe 21(3): 344-355.

38. Zhang Z, Han N, Shen Y (2020) S100A12 promotes inflammation and cell apoptosis in sepsis-induced ARDS via activation of NLRP3 inflammasome signaling. Mol Immunol 122: 38-48.

39. Schmmack G, Schorpp K, Kutzner K, Gehring T, Brenke JK, et al. (2017) TOD1/TRAF6 association balances p62dependent IL-1 signaling to NF-kB. eLife 6: e22416.

40. Dai T, Wu L, Wang S, Wang J, Xie F, et al. (2018) FAF1 regulates antiviral immunity by inhibition MAVS but is antagonized by phosphorylation upon viral infection. Cell Host Microbe 24(6): 776-790.

41. He TS, Chen T, Wang DD, Xu LG (2018) HAUS8 regulates RLR-VISA antiviral signaling positively by targeting VISA. Mol Med Rep 18(2): 2458-2466.

42. Bai Y, Yao L, Wei T (2020) Presumed asymptomatic carrier transmission of COVID-19. JAMA 323(14): 14061407.

43. Zhang R, Su H, Ma X, Xu X, Liang L, et al. (2019) MiRNA let-7b promotes the development of hypoxic pulmonary hypertension by targeting ACE2. Am J Physiol Lung Cell Mol Physiol 316(3): L547-L557.

44. Stenmark KR, Fagan KA, Frid MG (2006) Hypoxiainduced pulmonary vascular remodeling. Cellular and molecular mechanisms. Circ Res 99(7): 675-691.

45. Lei W, He Y, Shui X, Li G, Yan G, et al. (2016) Expression and analyses of the HIF-1 pathway in the lungs of human with pulmonary arterial hypertension. Mol Med Rep 14(5): 5383-4390.

46. Nicholson GT, Samai C, Kanaan U (2013) Pulmonary hypertension in Kawasaki disease. Pediatr Cardiol 34(8): 1966-1968.

47. Rong X, Ge D, Shen D, Chen X, Wang X, et al. (2018) miR-27b suppresses endothelial cell proliferation and migration by targeting Smad7 in Kawasaki disease. Cell Physiol Biochem 48(4): 1804-1814.

48. Zhang Y, Cao AL, Dong C (2017) Rs10719 polymorphism located within DROSHA 3'-untranslated region is responsible for development of primary hypertension by disrupting binding with microRNA-27b. Med Sci Monit 23: 911-918.

49. Wu D, Talbot Jr CC, Liu Q Jing ZC, Damico RL, et al. (2017)
Idnentifying microRNAs targeting Wnt/ $\beta$-catenin pathway in end-stage idiopathic pulmonary arterial hypertension. J Mol Med 94(8): 875-885.

50. Gelzer J, Kabat EA (1964) Specific fractionation of human antidextran antibodies. II. Assay of human antidextran sera and specifically fractionated purified antibodies by microcomplement fixation and complement fixation inhibition techniques. J Exp Med 119(6): 983-995.

51. Cheung AK, Henderon LW (1986) Effects of complement activation by hemodialysis membrane. Am J Nephrol 6(2): 81-91.

52. Duan K, Liu B, Li C, Zhang H, Yu T, et al. (2020) Effectiveness of convalescent plasma therapy in severe CIVID-19 patients. Proc Natl Acad Sci USA 117(17): 9490-9496.

53. Rosenberger CM, Podyminogin RL, NavarroG, Zhao GW, Askovich PS, et al. (2012) miR-451 regulates dendritic cell cytokine responses to influenza infection. J Immunol 189(12): 5965-5975.

54. Blane F, Furio L, Moisy D, Yen HL, Chignard M, et al. (2016) Targeting host calpain proteases decreases influenza A virus infection. Am J Physiol Lung Cell Mol Physiol 310(7): L689-L690.

55. Schneider M, Ackermann, K, Stuart M, Wex C, Protzer $\mathrm{U}$, et al. (2012) Severe acute respiratory syndrome coronavirus replication is severely impaired by MG132 due to proteasome-independent inhibition od M-calpain. J Virol 86(18): 10112-10122.

56. Bhatnagar T, Murhekar MV, Soneja M, Gupta N, Giri S, et al. (2020) Lopinavir/ritonavir combination therapy amongst symptomatic coronavirus disease 2019 patients in India: protocol for restricted public health emergency use. Indian J Med Res 151(2 \&3): 184-189.

57. Chen M, Du D, Zheng W, Liao M, Zhang L, et al. (2018) Small hepatitis delta antigen selectively binds to target mRNA in hepatic cells: a potential mechanism by which hepatitis D virus downregulates glutathione S-transferase P1 and induces liver injury and hepatocarcinogenesis. Biochem Cell Biol 97(2): 130-139.

58. Brisibe EA, Okada N, Mizukami H, Okuyama H, Fujii YR (2003) RNA interference: potential for the prevention of HIV infections and the challenging ahead. Trends Biotech 21(7): 306-311. 